\title{
Strong Convergence of an Iterative Method for Generalized Mixed Equilibrium Problems and Fixed Point Problems
}

\author{
Lijun Chen, Jianhua Huang* \\ Institute of Mathematics and Computer, Fuzhou University, Fuzhou, China \\ E-mail: chenlijun861010@163.com, fjihjh57@yahoo.com.cn \\ Received April 24, 2011; revised July 4, 2011; accepted July 11, 2011
}

\begin{abstract}
In this paper, we introduce a hybrid iterative method for finding a common element of the set of common solutions of generalized mixed equilibrium problems and the set of common fixed points of an finite family of nonexpansive mappings. Furthermore, we show a strong convergence theorem under some mild conditions.
\end{abstract}

Keywords: Generalized Mixed Equilibrium Problem, Hybrid Iterative Scheme, Fixed Point, Nonexpansive Mapping, Strong Convergence

\section{Introduction}

Equilibrium problems theory provides us with a natural, novel and unified framework for studying a wide class of problems arising in economics, finance, transportation, network and structural analysis, elasticity and optimization. The ideas and techniques of this theory are being used in a variety of diverse areas and proved to be productive and innovative.

Let $H$ be a Hilbert space with inner product $\langle\cdot, \cdot\rangle$ and norm $\|\cdot\|$. Let $C$ be a nonempty closed convex subset of $H$ and $T: C \rightarrow 2^{H}$ a multivalued mapping. Let $\phi: C \times C \rightarrow R$ be a real-valued function and $\Theta: H \times C \times C \rightarrow R$ be an equilibrium-like function, i.e., $\Theta(w, u, v)+\Theta(w, v, u)=0$ for each $(w, u, v) \in H \times C \times C$. The generalized mixed equilibrium problem (for short, GMEP) is to find $u \in C$ and $w \in T(u)$ such that

$$
\text { GMEP : } \Theta(w, u, v)+\phi(v, u)-\phi(u, u) \geq 0, \forall v \in C .
$$

in particular, if $T$ is single-valued mapping, this problem is equivalent to finding $u \in C$ such that

$$
\Theta(T(u), u, v)+\phi(v, u)-\phi(u, u) \geq 0, \forall v \in C .
$$

Denote the set of solutions of GMEP by $\Omega$.

Now, we recall the following definitions.

A mapping $f: C \rightarrow C$ is said to be contractive if there exists a constant $\alpha \in(0,1)$ such that $\|f(x)-f(y)\| \leq \alpha\|x-y\|$ for any $x, y \in C$. A mapping $g: C \rightarrow C$ is said to be firmly nonexpansive if $\|g(x)-g(y)\|^{2} \leq\langle g(x)-g(y), x-y\rangle$. A mapping $T: C \rightarrow C$ is said to be nonexpansive if $\|T x-T y\| \leq$ $\|x-y\|$ for any $x, y \in C$. The set of fixed points of $T$ is denoted by $F(T)$.

Let $\left\{T_{i}\right\}_{i=1}^{N}$ be a finite family of nonexpansive mappings of $C$ into $H$ and $\bigcap_{i=1}^{N} F\left(T_{i}\right) \neq \varnothing$. Define the mappings

$$
\left\{\begin{array}{l}
U_{n, 1}=\lambda_{n, 1} T_{1}+\left(1-\lambda_{n, 1}\right) I \\
U_{n, 2}=\lambda_{n, 2} T_{2} U_{n, 1}+\left(1-\lambda_{n, 2}\right) I \\
\vdots \\
U_{n, N-1}=\lambda_{n, N-1} T_{N-1} U_{n, N-2}+\left(1-\lambda_{n, N-1}\right) I \\
W_{n}=U_{n, N}=\lambda_{n, N} T_{N} U_{n, N-1}+\left(1-\lambda_{n, N}\right) I
\end{array}\right.
$$

where $\left\{\lambda_{n, i}\right\}_{i=1}^{N} \subset(0,1]$ for all $n \geq 1$. Such a mapping $W_{n}$ is called $W$-mapping generated by $T_{1}, \cdots, T_{N}$ and $\left\{\lambda_{n, i}\right\}_{i=1}^{N}$.

\section{Preliminaries}

Let $C$ be a nonempty closed convex subset of a real Hilbert space $H$. Then, for any $x \in H$, there exists a unique nearest point in $C$, denoted by $P_{C}(x)$, such that

$$
\left\|x-P_{C}(x)\right\| \leq\|x-y\|
$$

for all $y \in C$. Such a $P_{C}$ is called the metric projection 
of $H$ into $C$. We know that $P_{C}$ is nonexpansive. What's more,

$$
\begin{gathered}
x^{*}=P_{C}(x) \Leftrightarrow\left\langle x-x^{*}, x^{*}-y\right\rangle \geq 0, \\
\forall y \in C .
\end{gathered}
$$

Let $C$ be a convex subset of a real Hilbert space $H$, $\eta: C \times C \rightarrow H$ and $k: C \rightarrow R$ a Frechet differential function. Then $k$ is said to be $\eta$-strongly convex if there exists a constant $\mu>0$ such that

$$
\begin{gathered}
k(y)-k(x)-\left\langle k^{\prime}(x), \eta(y, x)\right\rangle \geq \frac{\mu}{2}\|x-y\|^{2}, \\
\forall x, y \in C .
\end{gathered}
$$

If $\mu=0$, then $k$ is said to be $\eta$-convex. In particular, if $\eta(y, x)=y-x$ for all $y, x \in C$, then $k$ is said to be strongly convex.

Let $C$ be a nonempty subset of a real Hilbert space $H$. A bifunction $\phi(\cdot, \cdot): C \times C \rightarrow R$ is said to be skewsymmetric if

$$
\begin{gathered}
\phi(u, v)+\phi(v, u)-\phi(u, u)-\phi(v, v) \leq 0, \\
\forall u, v \in C .
\end{gathered}
$$

It is easy to see that if the skew-symmetric bifunction $\phi(\cdot, \cdot)$ is linear in both arguments, then

$$
\phi(u, v) \geq 0, \forall u \in C .
$$

We denote $\rightarrow$ for weak convergence and $\rightarrow$ for strong convergence. A bifunction $\phi: C \times C \rightarrow R$ is called weakly sequentially continuous at $\left(x_{0}, y_{0}\right) \in C \times C$ if $\phi\left(x_{n}, y_{n}\right) \rightarrow \phi\left(x_{0}, y_{0}\right)$ as $n \rightarrow \infty$ for each sequence $\left\{\left(x_{n}, y_{n}\right)\right\}$ in $C \times C$ converging weakly to $\left(x_{0}, y_{0}\right)$. The function $\phi(\cdot, \cdot)$ is called weakly sequentially continuous on $C \times C$ if it is weakly sequentially continuous at each point of $C \times C$.

Let $C B(X)$ denote the set of nonempty closed bounded subset of $X$. For $A, B \in C B(X)$, define the Hausdorff metric $\hbar$ as follows:

$$
\begin{aligned}
\hbar(A, B)= & \max \left\{\sup _{a \in A} \inf f_{b \in B} d(a, b),\right. \\
& \left.\sup _{b \in A} i n f_{a \in B} d(b, a)\right\} .
\end{aligned}
$$

In order to solve the generalized mixed equilibrium problems for an equilibrium-like bifunction
$\Theta: H \times C \times C \rightarrow R$, we assume that $\Theta$ satisfies the following conditions with respect to the multivalued mapping $T: C \rightarrow 2^{H}$ :

$\left(\Theta_{1}\right)$ for each fixed $v \in C,(w, u) \mapsto \Theta(w, u, v)$ is an upper semicontinuous function from $H \times C$ to $R$, that is, $w_{n} \rightarrow w$ and $u_{n} \rightarrow u$ imply

$\limsup _{n \rightarrow \infty} \Theta\left(w_{n}, u_{n}, v\right) \leq \Theta(w, u, v)$;

$\left(\Theta_{2}\right)$ for each fixed $(w, v) \in H \times C, u \mapsto \Theta(w, u, v)$ is a concave function;

$\left(\Theta_{3}\right)$ for each fixed $(w, u) \in H \times C, \quad v \mapsto \Theta(w, u, v)$ is a convex function;

$$
\begin{gathered}
\left(\Theta_{4}\right) \Theta\left(w_{1}, T_{r}(x), T_{s}(y)\right)+\Theta\left(w_{2}, T_{s}(y), T_{r}(x)\right) \\
\leq-\gamma\left\|T_{r}(x)-T_{s}(y)\right\|^{2}
\end{gathered}
$$

for all $x, y \in C$ and $r, s \in(0, \infty)$, where $\gamma>0, w_{1} \in T(x)$ and $w_{2} \in T(y)$.

Let $k: C \rightarrow R$ be a differential function with Frechet derivative $k^{\prime}(x)$ at $x$ satisfying the following:

$\left(k_{1}\right) \quad k^{\prime}$ is continuous from the weak topology to the strong topology;

$\left(k_{2}\right) k^{\prime}$ is Lipschitz continuous with constant $v>0$.

Let $\eta: C \times C \rightarrow H$ be a function satisfying the following:

$\left(\eta_{1}\right) \quad \eta(x, y)+\eta(y, x)=0$ for all $x, y \in C$;

$\left(\eta_{2}\right) \quad \eta(\cdot, \cdot)$ is affine in the first coordinate variable;

$\left(\eta_{3}\right)$ for each fixed $y \in C, y \in C x \mapsto \eta(y, x)$ is sequentially continuous from the weak topology to the weak topology.

Let $C$ be a nonempty closed convex subset of a real Hilbert space and $T: C \rightarrow 2^{H}$ a multivalued mapping. For $x \in C$, let $w \in T(x)$. Let $\phi: C \rightarrow R$ be a realvalued function satisfying the following:

$\left(\phi_{1}\right) \phi(\cdot, \cdot)$ is skew symmetric;

$\left(\phi_{2}\right)$ for each fixed $y \in C, \phi(\cdot, y)$ is convex and upper semicontinuous;

$\left(\phi_{3}\right) \phi(\cdot, \cdot)$ is weakly continuous on $C \times C$.

Recently Wei-You Zeng, Nan-Jing Huang and ChangWen Zhao [1] introduce and consider a new class of equilibrium problems, which is known as the generalized mixed equilibrium problems. Furthermore, they introduce an iterative scheme (1.4) by the viscosity approximation method for finding a common element of the set of common solutions for generalized mixed equilibrium problems and the set of common fixed points of a sequence of nonexpansive mappings in Hilbert space.

$$
\left\{\begin{array}{l}
\left\|w_{n}-w_{n+1}\right\| \leq\left(1+\frac{1}{n}\right) \hbar\left(T\left(x_{n}\right), T\left(x_{n+1}\right)\right. \\
\Theta\left(w_{n}, u_{n}, v\right)+\phi\left(v, u_{n}\right)-\phi\left(u_{n}, u_{n}\right)+\frac{1}{r}\left\langle k^{\prime}\left(u_{n}\right)-k^{\prime}\left(x_{n}\right), \eta\left(v, u_{n}\right)\right\rangle \geq 0, \forall v \in C \\
x_{n+1}=\alpha_{n} f\left(x_{n}\right)+\left(1-\alpha_{n}\right) W_{n} u_{n}
\end{array}\right.
$$


Motivated and inspired by the research going on in this important field, we introduce the following hybrid iterative scheme (1.5) for finding a common element of the set of common solutions for generalized mixed equilibrium problems and the set of common fixed points of a sequence of nonexpansive mappings. We show that the approximation solution converges strongly to a unique solution of a class of variational inequalities under some mild conditions. Results obtained in this paper can be viewed as an improvement and refinement of the recent results in this direction.

Algorithm 1.1. Let $C$ be a nonempty closed convex subset of a real Hilbert space $H, T: C \rightarrow C B(H)$ be a multivalued mapping, $f$ be a contraction of $C$ into itself with coefficient $\alpha \in(0,1)$. Let $W_{n}: C \rightarrow C$ be defined by (1.3), and $r>0$. For given $x_{1} \in C$ and $w_{1} \in T\left(x_{1}\right)$, there exists sequences $\left\{x_{n}\right\},\left\{u_{n}\right\}$ in $C$ and $\left\{w_{n}: w_{n} \in T\left(x_{n}\right)\right\}$ in $H$ such that for all $n=1,2, \cdots$,

$$
\left\{\begin{array}{l}
\left\|w_{n}-w_{n+1}\right\| \leq\left(1+\frac{1}{n}\right) \hbar\left(T\left(x_{n}\right), T\left(x_{n+1}\right) ;\right. \\
\Theta\left(w_{n}, u_{n}, v\right)+\phi\left(v, u_{n}\right)-\phi\left(u_{n}, u_{n}\right)+\frac{1}{r}\left\langle k^{\prime}\left(u_{n}\right)-k^{\prime}\left(x_{n}\right), \eta\left(v, u_{n}\right)\right\rangle \geq 0, \forall v \in C \\
x_{n+1}=a_{n} f\left(W_{n} x_{n}\right)+b_{n} x_{n}+c_{n} W_{n} u_{n}
\end{array}\right.
$$

where $\left\{a_{n}\right\},\left\{b_{n}\right\}$ and $\left\{c_{n}\right\}$ are three sequences in $(0$, 1) such that $a_{n}+b_{n}+c_{n}=1$.

It is easy to see that the iterative scheme (1.5) may be well defined.

Let $r$ be a positive number. For a given point $x \in C$ and $w_{x} \in T(x)$, consider the following auxiliary problem for GMEP: find $u \in C$ such that

$$
\begin{gathered}
\Theta\left(w_{x}, u, v\right)+\phi(v, u)-\phi(u, u) \\
+\frac{1}{r}\left\langle k^{\prime}(u)-k^{\prime}(x), \eta(v, u)\right\rangle \geq 0, \forall v \in C,
\end{gathered}
$$

It is easy to see that if $u=x$, then $u$ is a solution of GMEP.
We need the following important results.

Lemma 1.1. [2] Let $C$ be a nonempty closed convex bounded subset of a real Hilbert space $H$ and let $\phi: C \times C \rightarrow R$ be a real-valued function satisfying $\left(\phi_{1}\right)$ $\left(\phi_{3}\right)$. Let $T: C \rightarrow 2^{H}$ be a multivalued mapping and $\Theta: H \times C \times C \rightarrow R$ be an equilibrium-like bifunction satisfying the conditions $\left(\Theta_{1}\right)-\left(\Theta_{4}\right)$. Assume that $\eta: C \times C \rightarrow H$ is a Lipschitz function with lipschitz constant $\lambda>0$ which satisfies the conditions $\left(\eta_{1}\right)$ $\left(\eta_{3}\right)$. Let $k: C \rightarrow R$ be an $\eta$-strongly convex function with constant $\mu>0$ which satisfies the conditions $\left(k_{1}\right)$ and $\left(k_{2}\right)$. For each $x \in C$, let $w_{x} \in T(x)$. For $r>0$, define a mapping $T_{r}: C \rightarrow C$ by

$$
T_{r}(x)=\left\{u \in C: \Theta\left(w_{x}, u, v\right)+\phi(v, u)-\phi(u, u)+\frac{1}{r}\left\langle k^{\prime}(u)-k^{\prime}(x), \eta(v, u)\right\rangle \geq 0, \forall v \in C\right\}
$$

Then there hold the following:

1) the auxiliary problem (1.6) has a unique solution;

2) $T_{r}$ is single-valued;

3) if $\lambda v / \mu \leq 1$, it follows that $T_{r}$ is firmly nonexpansive;

4) $F\left(T_{r}\right)=\Omega$;

5) $\Omega$ is closed and convex.

Lemma 1.2. [3] Let $H$ be a real Hilbert space and let $C$ be a nonempty closed convex subset of $H$. Let
$\left\{T_{i}\right\}_{i=1}^{N}$ be a finite family of nonexpansive mappings of $C$ into $H$ and $\bigcap_{i=1}^{N} F\left(T_{i}\right) \neq \varnothing$, and let $\left\{\lambda_{n, i}\right\}_{i=1}^{N}$ be a sequence in $(0, b]$ for some $b \in(0,1)$. Then, $F\left(W_{n}\right)=\bigcap_{i=1}^{N} F\left(T_{i}\right)$.

Lemma 1.3. [4] If the sequences $\left\{u_{n}\right\}$ and $\left\{x_{n}\right\}$ are bounded and $W_{n}$ is defined by (1.3), then the following estimates hold:

$$
\left\|W_{n+1} u_{n+1}-W_{n} u_{n}\right\| \leq\left\|u_{n+1}-u_{n}\right\|+2 M \sum_{i=1}^{N}\left|\lambda_{n+1, i}-\lambda_{n, i}\right|, \quad \forall n \geq 0
$$

and

$$
\left\|W_{n+1} x_{n+1}-W_{n} x_{n}\right\| \leq\left\|x_{n+1}-x_{n}\right\|+2 M \sum_{i=1}^{N}\left|\lambda_{n+1, i}-\lambda_{n, i}\right|, \quad \forall n \geq 0
$$


for some constant $M>0$.

Lemma 1.4. [4] In a real Hilbert space $H, \forall x, y, z \in H$ and $t_{1}, t_{2}, t_{3} \in[0,1]$ with $t_{1}+t_{2}+t_{3}=1$, there holds the following equality:

$$
\left\|t_{1} x+t_{2} y+t_{3} z\right\|^{2} \leq t_{1}\|x\|^{2}+t_{2}\|y\|^{2}+t_{3}\|z\|^{2} .
$$

Lemma 1.5. [6] Let $\left\{x_{n}\right\}$ and $\left\{u_{n}\right\}$ be bounded sequences in a Banach space $X$ and let $\left\{b_{n}\right\}$ be a sequence in $[0,1]$ with $0<\liminf _{n \rightarrow \infty} b_{n} \leq \limsup _{n \rightarrow \infty} b_{n}<1$. Suppose

$$
x_{n+1}=\left(1-b_{n}\right) z_{n}+b_{n} x_{n}
$$

for all integers $n \geq 0$ and

$$
\limsup _{n \rightarrow \infty}\left(\left\|z_{n+1}-z_{n}\right\|-\left\|x_{n+1}-x_{n}\right\|\right) \leq 0 \text {. }
$$

Then,

$$
\lim _{n \rightarrow \infty}\left\|z_{n}-x_{n}\right\|=0
$$

Lemma 1.6. [5] Let $\left\{a_{n}\right\}$ is a sequence of nonnegative real numbers such that

$$
a_{n+1} \leq\left(1-\delta_{n}\right) a_{n}+b_{n}, \forall n=1,2, \cdots
$$

where $\left\{\delta_{n}\right\}$ is a sequence in $(0,1), \quad \sum_{n=1}^{\infty} \delta_{n}=\infty$ and $\limsup _{n \rightarrow \infty} b_{n} / \delta_{n} \leq 0$, then $\lim _{n \rightarrow \infty} a_{n}=0$.

Lemma 1.7. [2] Let $\left\{x_{n}\right\}$ be a sequence in a normed space $(X,\|\cdot\|)$ such that

$$
\left\|x_{n+1}-x_{n+2}\right\| \leq \theta\left\|x_{n}-x_{n+1}\right\| s_{n}+r_{n}, \forall n=1,2, \cdots
$$

where $\theta \in(0,1)$, and $\left\{s_{n}\right\}$ and $\left\{r_{n}\right\}$ are sequences satisfying the following conditions:

1) $s_{n} \geq 1$ and $\sum_{n=1}^{\infty}\left(s_{n}-1\right)<\infty$;

2) $r_{n} \geq 0$, and $\sum_{n=1}^{\infty} r_{n}<\infty$.

Then $\left\{x_{n}\right\}$ is a Cauchy sequence.

Lemma 1.8. [7] Let $A, B \in C B(X)$ and $a \in A$. Then for $\rho>1$, there must exist a point $b \in B$ such that $d(a, b) \leq \rho \hbar(A, B)$.

Lemma 1.9. [5] In a real Hilbert space $H$, there holds the following equality:

$$
\|x+y\|^{2} \leq\|x\|^{2}+2\langle y, x+y\rangle, \forall x, y \in H .
$$

\section{Main Results}

Theorem 2.1. Let $C$ be a nonempty closed convex bounded subset of a real Hilbert space $H$ and $r>0$, $T: C \rightarrow C B(H)$ be a multivalued $\hbar$-Lipschitz continuous mapping with constant $L>0$, and let $\phi: C \times C \rightarrow R$ be a real-valued function satisfying $\left(\phi_{1}\right)-\left(\phi_{3}\right)$. and $\Theta: H \times C \times C \rightarrow R$ be an equilibrium-like function satisfying the conditions $\left(\Theta_{1}\right)-\left(\Theta_{4}\right)$. Assume that $\eta: C \times C \rightarrow H$ is a Lipschitz function with lipschitz constant $\lambda>0$ which satisfies the conditions $\left(\eta_{1}\right)$ $\left(\eta_{3}\right)$. Let $k: C \rightarrow R$ be an $\eta$-strongly convex function with constant $\mu>0$ which satisfies the conditions $\left(k_{1}\right)$ and $\left(k_{2}\right)$. with $\lambda v / \mu \leq 1$. Let $\left\{T_{i}\right\}_{i=1}^{N}$ be a finite family of nonexpansive mappings on $H$ such that $\bigcap_{i=1}^{N} F\left(T_{i}\right) \cap \Omega \neq \varnothing$. Let $f$ be a contraction of $C$ into itself with coefficient $\alpha \in(0,1)$. Let $\left\{x_{n}\right\},\left\{u_{n}\right\},\left\{w_{n}\right\}$ be sequences generated by (1.5), where $\left\{a_{n}\right\},\left\{b_{n}\right\}$ and $\left\{c_{n}\right\}$ are three sequences in $(0,1)$ with $a_{n}+b_{n}+c_{n}=1$ satisfying the following conditions:

1) $\lim _{n \rightarrow \infty} a_{n}=0, \sum_{n=1}^{\infty} a_{n}=\infty$ and $\sum_{n=1}^{\infty}\left|a_{n+1}-a_{n}\right|<\infty$;

2) $0<\liminf _{n \rightarrow \infty} b_{n} \leq \limsup _{n \rightarrow \infty} b_{n}<1$ and $\sum_{n=1}^{\infty}\left|b_{n+1}-b_{n}\right|<\infty$;

3) $\sum_{i=1}^{N}\left|\lambda_{n+1, i}-\lambda_{n, i}\right|<\infty$;

4) $\sum_{n=1}^{\infty}\left|c_{n+1}-c_{n}\right|<\infty$.

Then the sequences $\left\{x_{n}\right\}$ and $\left\{u_{n}\right\}$ converge strongly to $x^{*} \in \cap_{i=1}^{N} F\left(T_{i}\right) \cap \Omega$, and $\left\{w_{n}\right\}$ converges strongly to $w^{*} \in T\left(x^{*}\right)$, where $x^{*}=P_{\cap_{i=1}^{N} F\left(T_{i}\right) \cap \Omega} f\left(x^{*}\right)$.

To proof Theorem 2.1, we first establish the following lemma.

Lemma 2.1. Let $C$ be a nonempty closed convex bounded subset of a real Hilbert space $H$ and $r>0$, $T: C \rightarrow C B(H)$ be a multivalued $\hbar$-Lipschitz continuous mapping with constant $L>0$, and let $\phi: C \times C \rightarrow R$ be a real-valued function satisfying $\left(\phi_{1}\right)-\left(\phi_{3}\right)$. and $\Theta: H \times C \times C \rightarrow R$ be an equilibrium-like function satisfying the conditions $\left(\Theta_{1}\right)-\left(\Theta_{4}\right)$. Assume that $\eta: C \times C \rightarrow H$ is a Lipschitz function with lipschitz constant $\lambda>0$ which satisfies the conditions $\left(\eta_{1}\right)$ $\left(\eta_{3}\right)$. Let $k: C \rightarrow R$ be an $\eta$-strongly convex function with constant $\mu>0$ which satisfies the conditions $\left(k_{1}\right)$ and $\left(k_{2}\right)$ with $\lambda v / \mu \leq 1$. Let $\left\{T_{i}\right\}_{i=1}^{N}$ be a finite family of nonexpansive mappings on $H$ such that $\bigcap_{i=1}^{N} F\left(T_{i}\right) \cap \Omega \neq \varnothing$. Let $f$ be a contraction of $C$ into itself with coefficient $\alpha \in(0,1)$. Let $\left\{x_{n}\right\},\left\{u_{n}\right\},\left\{w_{n}\right\}$ be sequences generated by (1.5), where $\left\{a_{n}\right\},\left\{b_{n}\right\}$ and $\left\{c_{n}\right\}$ are three sequences in $(0,1)$ with $a_{n}+b_{n}+c_{n}=1$, satisfying the following conditions:

1) $\lim _{n \rightarrow \infty} a_{n}=0, \sum_{n=1}^{\infty} a_{n}=\infty$ and $\sum_{n=1}^{\infty}\left|a_{n+1}-a_{n}\right|<\infty$;

2) $0<\liminf _{n \rightarrow \infty} b_{n} \leq \limsup _{n \rightarrow \infty} b_{n}<1$;

3) $\lim _{n \rightarrow \infty}\left|\lambda_{n, i+1}-\lambda_{n, i}\right|=0$;

4) $\sum_{n=1}^{\infty}\left|c_{n+1}-c_{n}\right|<\infty$. then

1) $\lim _{n \rightarrow \infty}\left\|u_{n+1}-u_{n}\right\|=0, \lim _{n \rightarrow \infty}\left\|x_{n+1}-x_{n}\right\|=0$;

2) $\lim _{n \rightarrow \infty}\left\|x_{n}-W_{n} u_{n}\right\|=0, \lim _{n \rightarrow \infty}\left\|x_{n}-u_{n}\right\|=0$.

Proof. 1) From the nonexpansity of $T_{r}$, we have 


$$
\left\|u_{n+1}-u_{n}\right\|=\left\|T_{r} x_{n+1}-T_{r} x_{n}\right\| \leq\left\|x_{n+1}-x_{n}\right\|
$$

and set $z_{n}=\frac{x_{n+1}-b_{n} x_{n}}{1-b_{n}}$, we obtain

$$
\begin{aligned}
z_{n+1}-Z_{n}= & \frac{x_{n+2}-b_{n+1} x_{n+1}}{1-b_{n+1}}-\frac{x_{n+1}-b_{n} x_{n}}{1-b_{n}}=\frac{a_{n+1} f\left(W_{n+1} x_{n+1}\right)+c_{n+1} W_{n+1} u_{n+1}}{1-b_{n+1}}-\frac{a_{n} f\left(W_{n} x_{n}\right)+c_{n} W_{n} u_{n}}{1-b_{n}} \\
= & \frac{a_{n+1}}{1-b_{n+1}}\left[f\left(W_{n+1} x_{n+1}\right)-f\left(W_{n} x_{n}\right)\right]+\left(\frac{a_{n+1}}{1-b_{n+1}}-\frac{a_{n}}{1-b_{n}}\right) f\left(W_{n} x_{n}\right) \\
& +\frac{c_{n+1}}{1-b_{n+1}}\left(W_{n+1} u_{n+1}-W_{n} u_{n}\right)+\left(\frac{c_{n+1}}{1-b_{n+1}}-\frac{c_{n}}{1-b_{n}}\right) W_{n} u_{n}
\end{aligned}
$$

By Lemma 1.3, we arrive at

$$
\begin{aligned}
\left\|z_{n+1}-z_{n}\right\| \leq & \frac{a_{n+1}}{1-b_{n+1}}\left\|f\left(W_{n+1} x_{n+1}\right)-f\left(W_{n} x_{n}\right)\right\|+\left|\frac{a_{n+1}}{1-b_{n+1}}-\frac{a_{n}}{1-b_{n}}\right|\left(\left\|f\left(W_{n} x_{n}\right)\right\|+\left\|W_{n} u_{n}\right\|\right)+\frac{c_{n+1}}{1-b_{n+1}}\left\|W_{n+1} u_{n+1}-W_{n} u_{n}\right\| \\
\leq & \frac{\alpha a_{n+1}}{1-b_{n+1}}\left[\left\|x_{n+1}-x_{n}\right\|+2 M \sum_{i=1}^{N}\left|\lambda_{n+1, i}-\lambda_{n, i}\right|\right]+\mid \frac{a_{n+1}}{1-b_{n+1}}-\frac{a_{n}}{1-b_{n}}\left(\left\|f\left(W_{n} x_{n}\right)\right\|+\left\|W_{n} u_{n}\right\|\right) \\
& +\frac{c_{n+1}}{1-b_{n+1}}\left[\left\|u_{n+1}-u_{n}\right\|+2 M \sum_{i=1}^{N}\left|\lambda_{n+1, i}-\lambda_{n, i}\right|\right]
\end{aligned}
$$

Hence, it follows from (2.1) that

$$
\begin{aligned}
\left\|z_{n+1}-z_{n}\right\| \leq & \frac{\alpha a_{n+1}}{1-b_{n+1}}\left[\left\|x_{n+1}-x_{n}\right\|+2 M \sum_{i=1}^{N}\left|\lambda_{n+1, i}-\lambda_{n, i}\right|\right]+\left|\frac{a_{n+1}}{1-b_{n+1}}-\frac{a_{n}}{1-b_{n}}\right|\left(\left\|f\left(W_{n} x_{n}\right)\right\|+\left\|W_{n} u_{n}\right\|\right) \\
& +\frac{c_{n+1}}{1-b_{n+1}}\left[\left\|x_{n+1}-x_{n}\right\|+2 M \sum_{i=1}^{N}\left|\lambda_{n+1, i}-\lambda_{n, i}\right|\right] \\
\leq & \left\|x_{n+1}-x_{n}\right\|+\left|\frac{a_{n+1}}{1-b_{n+1}}-\frac{a_{n}}{1-b_{n}}\right|\left(\left\|f\left(W_{n} x_{n}\right)\right\|+\left\|W_{n} u_{n}\right\|\right)+2 M \sum_{i=1}^{N}\left|\lambda_{n+1, i}-\lambda_{n, i}\right|
\end{aligned}
$$

It follows from conditions (a) and (c), we have

$$
\limsup _{n \rightarrow \infty}\left(\left\|z_{n+1}-z_{n}\right\|-\left\|x_{n+1}-x_{n}\right\|\right) \leq 0 .
$$

Hence by Lemma 1.5, we can see that

$$
\lim _{n \rightarrow \infty}\left\|z_{n}-x_{n}\right\|=0
$$

Consequently

$$
\lim _{n \rightarrow \infty}\left\|x_{n+1}-x_{n}\right\|=\lim _{n \rightarrow \infty}\left(1-b_{n}\right)\left\|z_{n}-x_{n}\right\|=0
$$

From (2.1), we get

$$
\lim _{n \rightarrow \infty}\left\|u_{n+1}-u_{n}\right\|=0
$$

2) In view of (1.5), we conclude that

$$
\begin{aligned}
\left\|x_{n}-W_{n} u_{n}\right\| \leq & \left\|x_{n}-x_{n+1}\right\|+\left\|x_{n+1}-W_{n} u_{n}\right\| \\
\leq & \left\|x_{n}-x_{n+1}\right\|+a_{n}\left\|f\left(W_{n} x_{n}\right)-W_{n} u_{n}\right\| \\
& +b_{n}\left\|x_{n}-W_{n} u_{n}\right\|,
\end{aligned}
$$

that is

$$
\begin{aligned}
\left\|x_{n}-W_{n} u_{n}\right\| \leq & \frac{1}{1-b_{n}}\left\|x_{n}-x_{n+1}\right\| \\
& +\frac{a_{n}}{1-b_{n}}\left\|f\left(W_{n} x_{n}\right)-W_{n} u_{n}\right\|,
\end{aligned}
$$

which implies that

$$
\lim _{n \rightarrow \infty}\left\|x_{n}-W_{n} u_{n}\right\|=0
$$

For $p \in \Gamma=\cap_{i=1}^{N} F\left(T_{i}\right) \cap \Omega$, note that $T_{r}$ is firmly nonexpansive, we can see that

$$
\begin{aligned}
\left\|u_{n}-p\right\|^{2} & =\left\|T_{r} x_{n}-T_{r} p\right\|^{2} \leq\left\langle T_{r} x_{n}-T_{r} p, x_{n}-p\right\rangle \\
& =\left\langle u_{n}-p, x_{n}-p\right\rangle \\
& =\frac{1}{2}\left(\left\|u_{n}-p\right\|^{2}+\left\|x_{n}-p\right\|^{2}-\left\|u_{n}-x_{n}\right\|^{2}\right)
\end{aligned}
$$

and so

$$
\left\|u_{n}-p\right\|^{2} \leq\left\|x_{n}-p\right\|^{2}-\left\|u_{n}-x_{n}\right\|^{2} \leq\left\|x_{n}-p\right\|^{2}
$$

In view of Lemma 1.4, (2.6) and (2.7), we compute 
which follows that

$$
\begin{aligned}
\left\|x_{n+1}-p\right\|^{2} & \leq\left\|a_{n} f\left(W_{n} x_{n}\right)+b_{n} x_{n}+c_{n} W_{n} u_{n}-p\right\|^{2} \\
& \leq a_{n}\left\|f\left(W_{n} x_{n}\right)-p\right\|^{2}+b_{n}\left\|x_{n}-p\right\|^{2}+c_{n}\left\|W_{n} u_{n}-p\right\|^{2} \\
& \leq a_{n}\left\|f\left(W_{n} x_{n}\right)-p\right\|^{2}+b_{n}\left\|x_{n}-p\right\|^{2}+c_{n}\left\|u_{n}-p\right\|^{2} \\
& \leq a_{n}\left\|f\left(W_{n} x_{n}\right)-p\right\|^{2}+b_{n}\left\|x_{n}-p\right\|^{2}+c_{n}\left(\left\|x_{n}-p\right\|^{2}-\left\|x_{n}-u_{n}\right\|^{2}\right) \\
& \leq a_{n}\left\|f\left(W_{n} x_{n}\right)-p\right\|^{2}+\left\|x_{n}-p\right\|^{2}-c_{n}\left\|x_{n}-u_{n}\right\|^{2}
\end{aligned}
$$

$$
c_{n}\left\|x_{n}-u_{n}\right\|^{2} \leq\left(\left\|x_{n}-p\right\|+\left\|x_{n+1}-p\right\|\right)\left(\left\|x_{n+1}-x_{n}\right\|\right)+a_{n}\left\|f\left(W_{n} x_{n}\right)-p\right\|^{2}
$$

and hence

$$
\lim _{n \rightarrow \infty}\left\|x_{n}-u_{n}\right\|=0
$$

This completes the proof.

$$
\begin{aligned}
\left\|x_{n+1}-x_{n}\right\|= & \left\|\left[a_{n} f\left(W_{n} x_{n}\right)+b_{n} x_{n}+c_{n} W_{n} u_{n}\right]-\left[a_{n-1} f\left(W_{n-1} x_{n-1}\right)+b_{n-1} x_{n-1}+c_{n-1} W_{n-1} u_{n-1}\right]\right\| \\
\leq & \left|a_{n}-a_{n-1}\right| \cdot\left\|f\left(W_{n} x_{n}\right)\right\|+a_{n-1}\left\|f\left(W_{n} x_{n}\right)-f\left(W_{n-1} x_{n-1}\right)\right\|+\left|b_{n}-b_{n-1}\right| \cdot\left\|x_{n}\right\| \\
& +b_{n-1}\left\|x_{n}-x_{n-1}\right\|+\left|c_{n}-c_{n-1}\right| \cdot|| W_{n} u_{n}\left\|+c_{n-1}\right\| W_{n} u_{n}-W_{n-1} u_{n-1} \| \\
\leq & \left|a_{n}-a_{n-1}\right| \cdot\left\|f\left(W_{n} x_{n}\right)\right\|+a_{n-1} \alpha\left[\left\|x_{n}-x_{n-1}\right\|+2 M \sum_{i=1}^{N}\left|\lambda_{n+1, i}-\lambda_{n, i}\right|\right]+\left|b_{n}-b_{n-1}\right| \cdot \mid x_{n} \| \\
& +b_{n-1}\left\|x_{n}-x_{n-1}\right\|+\left|c_{n}-c_{n-1}\right| \cdot\left\|W_{n} u_{n}\right\|+c_{n-1}\left[\left\|u_{n}-u_{n-1}\right\|+2 M \sum_{i=1}^{N}\left|\lambda_{n+1, i}-\lambda_{n, i}\right|\right] \\
\leq & {\left[1-(1-\alpha) a_{n-1}\right]\left\|x_{n}-x_{n-1}\right\|+r_{n} }
\end{aligned}
$$

where $\theta_{n}=1-(1-\alpha) a_{n-1} \leq \frac{1}{2}, s_{n}=1$ and

$$
r_{n}=\left|a_{n}-a_{n-1}\right| \cdot|| f\left(W_{n} x_{n}\right)\left\|+\left|b_{n}-b_{n-1}\right| \cdot\right\| x_{n}\left\|+\left|c_{n}-c_{n-1}\right| \cdot\right\| W_{n} u_{n} \|+2 M \sum_{i=1}^{N}\left|\lambda_{n+1, i}-\lambda_{n, i}\right| \cdot
$$

By Lemma 1.7 and conditions (a)-(d), we conclude that $\left\{x_{n}\right\}$ is a Cauchy sequence in $C$ such that $\lim _{n \rightarrow \infty} u_{n}=x^{*}$, there exists an element $x^{*} \in C$. On the other hand, $\lim _{n \rightarrow \infty}\left\|x_{n}-u_{n}\right\|=0$ implies that $\lim _{n \rightarrow \infty} u_{n}=x^{*}$. From (1.5), we have

$$
\begin{aligned}
\left\|w_{n}-w_{n+1}\right\| & \leq\left(1+\frac{1}{n}\right) \hbar\left(T\left(x_{n}\right), T\left(x_{n+1}\right)\right) \\
& \leq 2 \hbar\left(T\left(x_{n}\right), T\left(x_{n+1}\right)\right) \leq 2 L\left\|x_{n}-x_{n+1}\right\|
\end{aligned}
$$

and for $m>n \geq 1$,

$$
\begin{aligned}
\| w_{m} & -w_{n}\left\|\leq \sum_{i=n}^{m-1}\right\| w_{i}-w_{i+1}\left\|\leq 2 L \sum_{i=n}^{m-1}\right\| x_{i}-x_{i+1} \| \\
\sum_{i=n}^{m-1} a_{i+1} & =\sum_{i=n}^{m-1}\left\|x_{i}-x_{i+1}\right\| \leq \sum_{i=n}^{m-1}\left(\theta a_{i}+r_{i}\right) \\
& =\theta \sum_{i=n}^{m-1} a_{i}+\sum_{i=n}^{m-1} r_{i} \leq \theta \sum_{i=n}^{m-1} a_{i+1}+\theta\left(a_{n}-a_{m}\right)+\sum_{i=n}^{m-1} r_{i} \\
& \leq \theta \sum_{i=n}^{m-1} a_{i+1}+\theta a_{n}+\sum_{i=n}^{m-1} r_{i}
\end{aligned}
$$

Hence

$$
\sum_{i=n}^{m-1}\left\|x_{i}-x_{i+1}\right\| \leq \frac{\theta}{1-\theta}\left\|x_{n}-x_{n-1}\right\|+\frac{\sum_{i=n}^{m-1} r_{i}}{1-\theta}
$$

In view of (2.4) and (2.8), we obtain

$$
\lim _{m, n \rightarrow \infty}\left\|w_{m}-w_{n}\right\|=0
$$

which implies that $\left\{w_{n}\right\}$ is a Cauchy sequence in $H$ and therefore there exists an element $w$ in $H$ such that $\lim _{n \rightarrow \infty} w_{n}=w$. Next we can see that

$$
\begin{aligned}
d\left(w, T\left(x^{*}\right)\right) & =\inf _{b \in T\left(x^{*}\right)} d(w, b) \leq\left\|w-w_{n}\right\|+d\left(w, T\left(x^{*}\right)\right) \\
& \leq\left\|w-w_{n}\right\|+\hbar\left(T\left(x_{n}\right), T\left(x^{*}\right)\right) \\
& \leq\left\|w-w_{n}\right\|+L\left\|x_{n}-x^{*}\right\|
\end{aligned}
$$

Hence, we derive that $d\left(w, T\left(x^{*}\right)\right)=0$, that is 
$w \in T\left(x^{*}\right)$ as $T\left(x^{*}\right) \in C B(H)$.

Step 2. Let $Q=P_{\cap_{i=1}^{N} F\left(T_{i}\right) \cap \Omega} f$. Then $Q$ is a contraction of $C$ into itself. In fact, for all $x, y \in C$

$$
\|Q(x)-Q(y)\| \leq\|f(x)-f(y)\| \leq \alpha\|x-y\|
$$

Therefore there exists a unique element $q \in C$ such that $q=Q(q)$. Noting that $q \in C$ and $Q(q) \in \cap_{i=1}^{N} F\left(T_{i}\right) \cap \Omega$, we get that $q \in \cap_{i=1}^{N} F\left(T_{i}\right) \cap \Omega$. Then

$$
\langle f(q)-q, p-q\rangle \leq 0, \forall p \in \cap_{i=1}^{N} F\left(T_{i}\right) \cap \Omega .
$$

Next, we show that $x^{*} \in \cap_{i=1}^{N} F\left(T_{i}\right) \cap \Omega$. Since $x_{n} \rightarrow x^{*}$ and $u_{n} \rightarrow x^{*}$, we know that $k^{\prime}\left(u_{n}\right)-k^{\prime}\left(x_{n}\right) \rightarrow 0$, From (1.5) and $\left(\Theta_{1}\right)$, we have

$$
\Theta\left(w, x^{*}, v\right)+\phi\left(v, x^{*}\right)-\phi\left(x^{*}, x^{*}\right) \geq 0
$$

that is $x^{*} \in \Omega$. We shall show $x^{*} \in F\left(W_{n}\right)$. Assume $x^{*} \notin F\left(W_{n}\right)$, that is $x^{*} \neq W_{n} x^{*}$. Since $\left\{u_{n}\right\}$ is bounded, there exists a subsequence $\left\{u_{n_{j}}\right\}$ of $\left\{u_{n}\right\}$ which converges weakly to $x^{*}$. By Lemma 2.1, we conclude that $\left\|W_{n} u_{n}-u_{n}\right\| \rightarrow 0$. From Opial's condition, we have

$$
\begin{aligned}
\liminf _{j \rightarrow \infty} \| u_{n_{j}} & -x^{*}\left\|<\liminf _{j \rightarrow \infty}\right\| u_{n_{j}}-W_{n} x^{*} \| \\
& \leq \liminf _{j \rightarrow \infty}\left(\left\|u_{n_{j}}-W_{n} u_{n_{j}}\right\|+\left\|W_{n} u_{n_{j}}-W_{n} x^{*}\right\|\right) \\
& \leq \liminf _{j \rightarrow \infty}\left\|u_{n_{j}}-x^{*}\right\|
\end{aligned}
$$

This is a contradiction. So, we get

$x^{*} \in F\left(W_{n}\right)=\cap_{i=1}^{N} F\left(T_{i}\right)$. Therefore $x^{*} \in \cap_{i=1}^{N} F\left(T_{i}\right) \cap \Omega$.

Step 3. From (2.13) and $x_{n} \rightarrow x^{*}$, we obtain

$$
\lim _{n \rightarrow \infty}\left\langle f(q)-q, x_{n}-q\right\rangle=\left\langle f(q)-q, x^{*}-q\right\rangle \leq 0
$$

By Lemma 1.9, (1.5) and (2.7), we compute

$$
\begin{aligned}
\left\|x_{n+1}-q\right\|^{2} & =\left\|a_{n}\left(f\left(W_{n} x_{n}\right)-q\right)+b_{n}\left(x_{n}-q\right)+c_{n}\left(W_{n} u_{n}-q\right)\right\|^{2} \\
& \leq\left\|b_{n}\left(x_{n}-q\right)+c_{n}\left(W_{n} u_{n}-q\right)\right\|^{2}+2 a_{n}\left\langle f\left(W_{n} x_{n}\right)-q, x_{n+1}-q\right\rangle \\
& \leq\left[b_{n}\left\|x_{n}-q\right\|+c_{n}\left\|u_{n}-q\right\|\right]^{2}+2 a_{n}\left\langle f\left(W_{n} x_{n}\right)-f(q), x_{n+1}-q\right\rangle+2 a_{n}\left\langle f(q)-q, x_{n+1}-q\right\rangle \\
& \leq\left[b_{n}\left\|x_{n}-q\right\|+c_{n}\left\|x_{n}-q\right\|\right]^{2}+2 \alpha a_{n}\left\|x_{n}-q\right\|\left\|x_{n+1}-q\right\|+2 a_{n}\left\langle f(q)-q, x_{n+1}-q\right\rangle
\end{aligned}
$$

Hence

$$
\begin{aligned}
\left\|x_{n+1}-q\right\|^{2} & \leq \frac{\left(1-a_{n}\right)^{2}+a_{n} \alpha}{1-a_{n} \alpha}\left\|x_{n}-q\right\|^{2}+\frac{2 a_{n}}{1-a_{n} \alpha}\left\langle f(q)-q, x_{n+1}-q\right\rangle \\
& =\left[\frac{1-2 a_{n}+\alpha a_{n}}{1-a_{n} \alpha}\right]\left\|x_{n}-q\right\|^{2}+\frac{a_{n}^{2}}{1-a_{n} \alpha}\left\|x_{n}-q\right\|^{2}+\frac{2 a_{n}}{1-a_{n} \alpha}\left\langle f(q)-q, x_{n+1}-q\right\rangle \\
& \leq\left[1-\frac{2(1-\alpha) a_{n}}{1-a_{n} \alpha}\right]\left\|x_{n}-q\right\|^{2}+\frac{2(1-\alpha) a_{n}}{1-a_{n} \alpha} \times\left\{\frac{a_{n} M_{1}}{2(1-\alpha)}+\frac{1}{1-\alpha}\left\langle f(q)-q, x_{n+1}-q\right\rangle\right\} \\
& =\left(1-\delta_{n}\right)\left\|x_{n}-q\right\|^{2}+\delta_{n} \sigma_{n},
\end{aligned}
$$

where $M_{1}=\sup \left\{\left\|x_{n}-q\right\|^{2}: n \geq 1\right\}, \delta_{n}=\frac{2(1-\alpha) a_{n}}{1-a_{n} \alpha}$ and $\sigma_{n}=\frac{a_{n} M_{1}}{2(1-\alpha)}+\frac{1}{1-\alpha}\left\langle f(q)-q, x_{n+1}-q\right\rangle$. It is easy to see that $\delta_{n} \rightarrow 0, \sum_{n=1}^{\infty} \delta_{n}=\infty$, and $\limsup _{n \rightarrow \infty} \sigma_{n} \leq 0$. Hence, by Lemma 1.6 , the sequence $\left\{x_{n}\right\}$ converges strongly to $q$. Consequently, we can obtain that $\left\{u_{n}\right\}$ also converges strongly to $q$, and so $x^{*}=q$. This completes the proof.

Putting $T_{i} x=x$ for all $i \geq 1$ in Theorem 2.1, we obtain.

Corollary 2.1. Let $C$ be a nonempty closed convex bounded subset of a real Hilbert space $H$,
$T: C \rightarrow C B(H)$ be a multivalued $\hbar$-Lipschitz continuous mapping with constant $L>0$, and let $\phi: C \times C \rightarrow R$ be a real-valued function satisfying $\left(\phi_{1}\right)-\left(\phi_{3}\right)$. and $\Theta: H \times C \times C \rightarrow R$ be an equilibrium-like function satisfying the conditions $\left(\Theta_{1}\right)-\left(\Theta_{4}\right)$ and $\Omega \neq \varnothing$. Assume that $\eta: C \times C \rightarrow H$ is a Lipschitz function with lipschitz constant $\lambda>0$ which satisfies the conditions $\left(\eta_{1}\right)-\left(\eta_{3}\right)$. Let $k: C \rightarrow R$ be an $\eta$-strongly convex function with constant $\mu>0$ which satisfies the conditions $\left(k_{1}\right)$ and $\left(k_{2}\right)$ with $\lambda v / \mu \leq 1$. Let $F$ be a contraction of $C$ into itself with coefficient $\alpha \in(0,1)$. Then the sequences $\left\{x_{n}\right\},\left\{u_{n}\right\}$, and $\left\{w_{n}\right\}$ generated iteratively by 


$$
\left\{\begin{array}{l}
\left\|w_{n}-w_{n+1}\right\| \leq\left(1+\frac{1}{n}\right) \hbar\left(T\left(x_{n}\right), T\left(x_{n+1}\right) ;\right. \\
\Theta\left(w_{n}, u_{n}, v\right)+\phi\left(v, u_{n}\right)-\phi\left(u_{n}, u_{n}\right)+\frac{1}{r}\left\langle k^{\prime}\left(u_{n}\right)-k^{\prime}\left(x_{n}\right), \eta\left(v, u_{n}\right)\right\rangle \geq 0, \forall v \in C \\
x_{n+1}=a_{n} f\left(x_{n}\right)+b_{n} x_{n}+c_{n} u_{n}
\end{array}\right.
$$

converge strongly to $x^{*} \in \Omega$, and $\left\{w_{n}\right\}$ converges strongly to $w^{*} \in T\left(x^{*}\right)$, where $x^{*}=P_{\Omega} f\left(x^{*}\right)$, and $\left\{a_{n}\right\},\left\{b_{n}\right\}$ and $\left\{c_{n}\right\}$ are sequences in $(0,1)$ with $a_{n}+b_{n}+c_{n}=1$, and $r>0$ satisfying the following conditions:

1) $\lim _{n \rightarrow \infty} a_{n}=0, \quad \sum_{n=1}^{\infty} a_{n}=\infty$ and $\sum_{n=1}^{\infty}\left|a_{n+1}-a_{n}\right|<\infty$;

2) $0<\liminf _{n \rightarrow \infty} b_{n} \leq \limsup _{n \rightarrow \infty} b_{n}<1$ and $\sum_{n=1}^{\infty}\left|b_{n+1}-b_{n}\right|<\infty$;

3) $\sum_{n=1}^{\infty}\left|c_{n+1}-c_{n}\right|<\infty$.

\section{References}

[1] W.-Y. Zeng, N.-J Huang and C.-W. Zhao, "Viscosity Approximation Methods for Generalized Mixed Equilibrium Problems and Fixed Points of a Sequence of Nonexpansive Mappings,” Fixed Point Theory Applications, Vol. 2008, 2008, Article ID 714939.

[2] D. R. Sahu, N.-C. Wong and J.-C. Yao, “On Convergence Analysis of an Iterative Algorithm for Finding Common Solution of Generalized Mixed Equilibrium Problems and Fixed Point Problems,” Mathematical Inequlities and
Applications, Article in Press.

[3] W. Takahashi and K. Shimoji, "Convergence Theorems for Nonexpansive Mappings and Feasibility Problems," Mathematical and Computer Modelling, Vol. 32, No. 11, 2000, pp. 1463-1471. doi:10.1016/S0895-7177(00)00218-1

[4] L.-C. Ceng and J.-C. Yao, "A Hybrid Iterative Scheme for Mixed Equilibrium Problems and Fixed Point Problems," Journal of Computational and Applied Mathematics, Vol. 214, No. 1, 2008, pp. 186-201. doi:10.1016/j.cam.2007.02.022

[5] H. K. Xu, "Viscosity Approximation for Nonexpansive Mappings," Journal of Mathematical Analysis and Applications, Vol. 298, No. 1, 2004, pp. 279-291. doi:10.1016/j.jmaa.2004.04.059

[6] T. Suzuki, "Strong Convergence of Krasnoselskii and Mann's Type Sequences for One-Parameter Nonexpansive Semigroups without Bochner Integrals," Journal of Mathematical Analysis and Applications, Vol. 305, No. 1, 2005, pp. 227-239. doi:10.1016/j.jmaa.2004.11.017

[7] S. B. Nadle Jr., "Multi-Valued Contraction Mappings," Pacific Journal of Mathematics, Vol. 30, 1969, pp. 475488. 